\title{
Shape Memory Properties of Biomedical Ti-Mo-Ag and Ti-Mo-Sn Alloys
}

\author{
Takashi Maeshima $^{1, *}$ and Minoru Nishida ${ }^{2}$ \\ ${ }^{1}$ Graduate School of Science and Technology, Kumamoto University, Kumamoto 860-8555, Japan \\ ${ }^{2}$ Department of Materials Science and Engineering, Kumamoto University, Kumamoto 860-8555, Japan
}

\begin{abstract}
Shape memory properties of Ti-Mo-Ag and Ti-Mo-Sn alloys consisting of biocompatible elements were investigated. The alloys with appropriate combination of Mo and $\mathrm{Ag}$ or Sn showed nearly perfect shape memory effect in convenient bending and heating tests. Recoverable tensile strain is more than $3 \%$ in some of Ti-Mo-Ag and Ti-Mo-Sn alloys. Microstructure observations and X-ray diffraction measurements before and after tensile test revealed that stress induced $\beta$ to $\alpha^{\prime \prime}$ martensitic transformation was origin of the shape memory effect.
\end{abstract}

(Received October 20, 2003; Accepted January 9, 2004)

Keywords: shape memory alloy, biomaterial, titanium-molybdenum-silver alloy, titanium-molybdenum-tin alloy, martensitic transformation

\section{Introduction}

Biomedical implant applications of Ti-Ni shape memory and superelastic alloys such as orthodontic wire, teeth-root prosthesis, stent in blood vessels ${ }^{1)}$ have been continuously increasing all over the world. However, it has been suspected that $\mathrm{Ni}$ is allergenic and carcinogenic to the human body. Any suspicion should be eliminated in the biomedical implant applications. Thus the development of Ni-free shape memory alloys has been urgently required. Recently, several Ni-free $\beta$-titanium shape memory and superelastic alloys such as Ti-Mo-Al, Ti-Mo-Ga and Ti-Nb-Sn have been developed along the above conception. ${ }^{2-4)}$ These ternary alloys are designed with appropriate combination of $\beta$ and $\alpha$ stabilizing elements. It is likely that there are lots of new combinations of such elements. In order to establish the biomedical implant applications of $\beta$-titanium shape memory and superelastic alloys, an extension of the candidate alloy systems is indispensable.

In the present study, Mo is selected as $\beta$ stabilizing element, and $\mathrm{Ag}$ and $\mathrm{Sn}$ is selected as the third element. All the elements indicate good biocompatibility. ${ }^{5)}$ Although Ag is classified as $\beta$ stabilizing element of $\beta$ eutectoid type, ${ }^{6)}$ the solubility limit to $\alpha$-Ti is about $4.7 \mathrm{~mol} \%$ at the $\alpha$ to $\beta$ transformation temperature, i.e., eutectoid temperature. This is the maximum among the other $\beta$ eutectoid type elements such as $\mathrm{Cr}, \mathrm{Mn}, \mathrm{Fe}, \mathrm{Ni}, \mathrm{Cu}$ and so on. In addition to the large solubility limit to $\alpha$-titanium the lowering of $\alpha$ to $\beta$ transformation temperature is about $27 \mathrm{~K}$, which is the minimum among the other $\beta$ eutectoid type elements. $\mathrm{Sn}$ is typical $\alpha$ stabilizing element of $\beta$ peritectoid type. ${ }^{6)}$ The purposes of the present study are to search the suitable composition to perform the shape memory effect in Ti-Mo$\mathrm{Ag}$ and Ti-Mo-Sn ternary alloys, and to characterize their shape memory properties.

\section{Experimental Procedure}

The nominal compositions of alloys investigated in the present study were listed in Table 1 . These alloys were arc melted in an Ar atmosphere using pure Ti (99.7 mass\%), pure
Table 1 List of nominal compositions of alloys prepared in the present study. + mark showing occurrence of shape memory effect with heating after convenient bending test.

\begin{tabular}{ccc}
\hline Ti-Mo $(\mathrm{mol} \%)$ & Ti-Mo-Ag $(\mathrm{mol} \%)$ & Ti-Mo-Sn $(\mathrm{mol} \%)$ \\
\hline Ti-5.0Mo + & Ti-5.0Mo-0.5Ag + & Ti-5.0Mo-1.0Sn + \\
Ti-6.0Mo + & Ti-5.0Mo-1.0Ag + & Ti-5.0Mo-2.0Sn + \\
& Ti-5.0Mo-2.0Ag + & Ti-5.0Mo-3.0Sn + \\
& Ti-5.0Mo-3.0Ag + & Ti-5.0Mo-4.0Sn + \\
& Ti-5.0Mo-4.0Ag + & Ti-5.0Mo-5.0Sn + \\
& Ti-5.0Mo-5.0Ag + & Ti-6.0Mo-1.0Sn + \\
& Ti-6.0Mo-1.0Ag + & Ti-6.0Mo-2.0Sn + \\
& Ti-6.0Mo-2.0Ag + & Ti-6.0Mo-3.0Sn + \\
& Ti-6.0Mo-3.0Ag + & Ti-6.0Mo-4.0Sn \\
& Ti-6.0Mo-4.0Ag + & Ti-6.0Mo-5.0Sn \\
\hline
\end{tabular}

Mo (99.95 mass\%), Ag (99.99 mass\%) and Sn (99.9 mass\%). In the preparation of $\mathrm{Ti}-\mathrm{Mo}-\mathrm{Ag}$ alloys, $\mathrm{Ti}$ and Mo were initially melted, and then $\mathrm{Ag}$ was added to Ti-Mo binary alloys. On the other hand, Ti, Mo and $\mathrm{Sn}$ were melted all together in the preparation of Ti-Mo-Sn alloys. Weight changes after arc melting were negligible small in both the alloys. The ingots were homogenized at $1373 \mathrm{~K}$ for $86.4 \mathrm{ks}$ in vacuum and then hot and cold rolled into plates of 0.3 to $0.5 \mathrm{~mm}$ in thickness. Specimens with prescribed shapes were prepared by spark machining from the rolled plates. They were encapsulated in an evacuated quartz tube, solutiontreated at $1273 \mathrm{~K}$ for $1.8 \mathrm{ks}$ and quenched into iced water with breaking the tube.

Shape memory properties were evaluated with convenient bending test and cyclic tensile test. The bending test was carried out as follows. The specimens were deformed in a round shape at room temperature. They were heated to about $550 \mathrm{~K}$ and then immediately quenched into ice water after the complement of shape recovery. The cyclic tensile and heating tests were performed in Instron-type machine with the initial strain rate of $4.16 \times 10^{-4} \mathrm{~s}^{-1}$. Phase constitution was identified by X-ray diffraction (XRD) at room temperature. The microstructures before and after tensile test were observed by optical microscopy. 
(a)

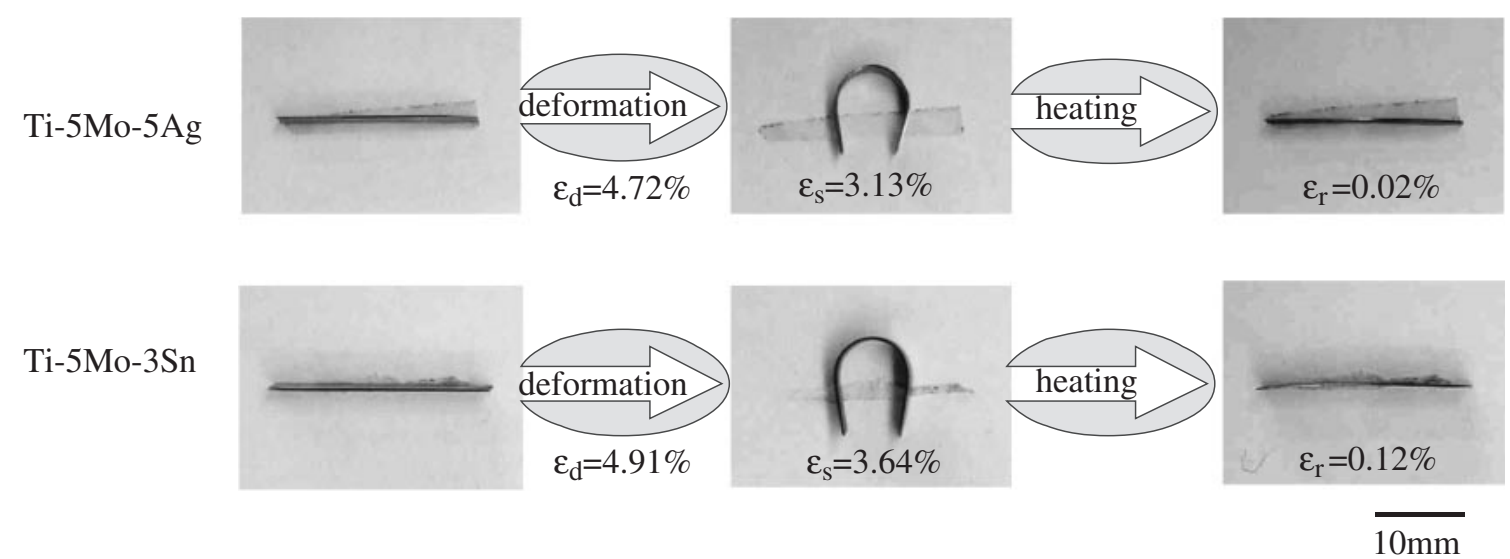

Fig. 1 Typical examples of convenient bending tests in (a) Ti-5Mo-5Ag and (b) Ti-5Mo-3Sn alloys.
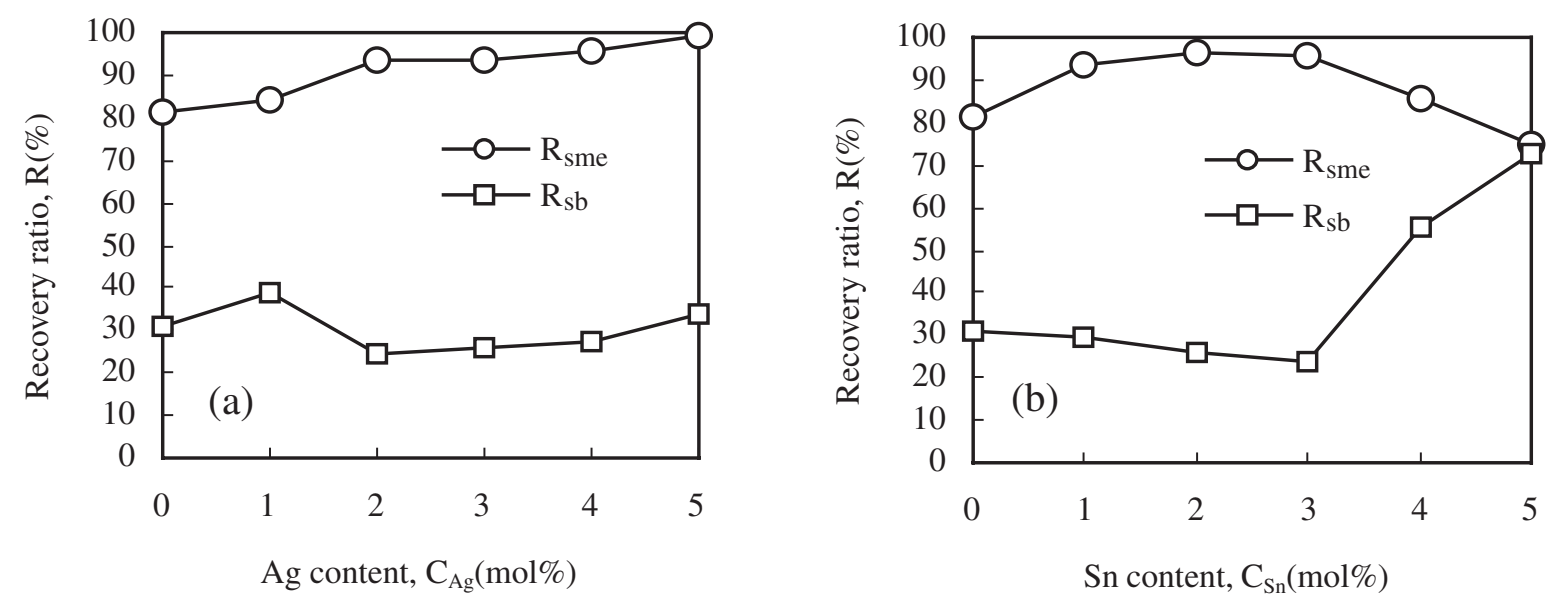

Fig. 2 Shape memory properties estimated from convenient bending test in (a) Ti-5Mo-Ag and (b) Ti-5Mo-Sn alloys.

\section{Results and Discussion}

The alloys prepared in the present study are listed in Table 1. In that table, the alloys marked ' + ' exhibit shape memory effect upon heating after the convenient bending test at room temperature. The incomplete shape memory effect is obtained even in the two binary alloys, i.e., Ti-5Mo and Ti$6 \mathrm{Mo}$ alloys. However, brittle fracture occurred in the binary alloys after two and three cycles of bending and recovery tests. There are two types of $\omega$ phase, i.e., athermal and isothermal ones, in $\beta$-titanium alloys. Generally, the former appears during cooling and the latter appears during aging. It is difficult to distinguish whether the athermal or the isothermal $\omega$ phases form during the bending and recovery test, because the test includes both aging and cooling processes as described above. However, since it is well known that the former has little influence on the mechanical properties and the latter causes the embrittlement, the brittle fracture in the binary alloys is mainly due to the isothermal $\omega$ phase formation during the bending and recovery tests. On the other hand, there is no brittle fracture in the ternary alloys after five cycles. The shape memory performance in Ti-5Mo based alloys was relatively superior to that in Ti-6Mo based alloys regardless of $\mathrm{Ag}$ or $\mathrm{Sn}$ addition. Therefore, we will describe the results of Ti-5Mo based alloys hereinafter. Figure 1 shows typical example of shape memory behavior in Ti-5Mo-Ag and Ti-5Mo-Sn alloys, respectively. It is clearly seen that both the Ti-5Mo-5Ag and Ti-5Mo-3Sn alloys exhibit nearly perfect shape memory effect. Shape memory properties in various Ti-5Mo-Ag and Ti-5Mo-Sn alloys estimated from the bending test are summarized in Figs. 2(a) and (b), respectively. Recovery ratio $R_{\text {sme }}$ with shape memory effect is defined as follows;

$$
R_{\mathrm{sme}}=\left(\varepsilon_{\mathrm{s}}-\varepsilon_{\mathrm{r}}\right) / \varepsilon_{\mathrm{s}} \times 100
$$

where $\varepsilon_{\mathrm{s}}$ : surface strain after bending deformation and $\varepsilon_{\mathrm{r}}$ : residual surface strain after heating as presented in Figs. 1(b) and (c), respectively. In addition to the $R_{\text {sme }}$, recovery ratio $R_{\mathrm{sb}}$ with spring back after bending deformation is also evaluated, since the $R_{\mathrm{sb}}$ gives a kind of potentiality that the specimen exhibits superelasiticity, as described below. The $R_{\mathrm{sb}}$ is given as follows;

$$
R_{\mathrm{sb}}=\left(\varepsilon_{\mathrm{d}}-\varepsilon_{\mathrm{s}}\right) / \varepsilon_{\mathrm{d}} \times 100
$$

where $\varepsilon_{\mathrm{d}}$ : applied surface strain with bending deformation as presented in Fig. 1. In Ti-5Mo-Ag system, the $R_{\text {sme }}$ increases 


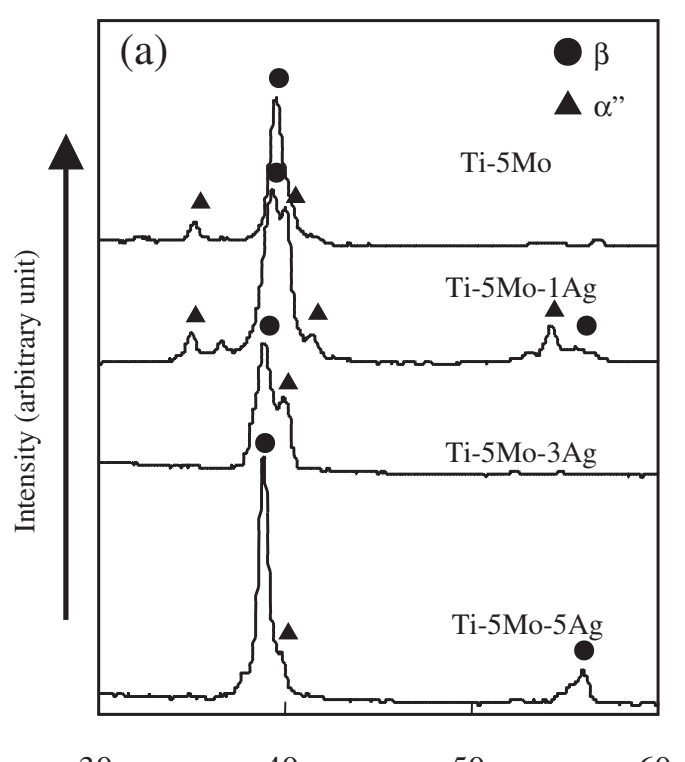

30

40

50

60

Diffraction angle, $2 \theta$

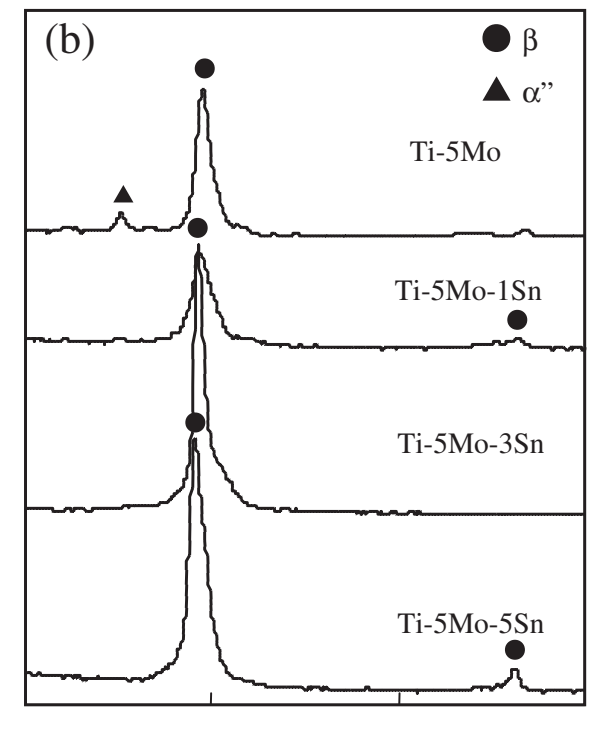

$40 \quad 50$

60

Fig. 3 XRD profile changes with Ag and Sn contents in (a) Ti-5Mo-Ag and (b) Ti-5Mo-Sn alloys, respectively.

with increasing Ag content and nearly perfect shape recovery is obtained in the Ti-5Mo-5Ag alloy. The $R_{\mathrm{sb}}$ is constantly around $30 \%$, irrespective of the $\mathrm{Ag}$ content. In Ti-5Mo-Sn system, the $R_{\text {sme }}$ increases with increasing Sn content up to $2 \%$ and then decreases gradually. The $R_{\mathrm{sb}}$ remarkably increases about 55 and $75 \%$ in Ti-5Mo-4 and 5Sn alloys, respectively. From these results, the additions of $\mathrm{Ag}$ and $\mathrm{Sn}$ improve the shape memory effect and suppress the isothermal $\omega$ phase in Ti-Mo alloy. Since $M_{\mathrm{s}}$ and $A_{\mathrm{s}}$ temperatures in each alloy could not be detected by DSC measurement, effects of $\mathrm{Ag}$ and $\mathrm{Sn}$ contents on the transformation behavior are not clear at present. $\mathrm{Ag}$ addition might have weak influence on the transformation temperature, since there are no drastic changes of $R_{\mathrm{sme}}$ and $R_{\mathrm{sb}}$ in Ti-Mo-Ag alloys. On the other hand, transformation temperatures in Ti-Mo-Sn alloys might decrease, since the $R_{\mathrm{sb}}$ increases with increasing Sn content of more than $4 \%$. This hypothesis is supported by tensile test at room temperature. The stress-strain curves of all the Ti5Mo-Ag alloys, and Ti-5Mo-1, 2 and 3Sn alloys showed typical shape memory type behavior as later presented in Fig. 5, i.e., there is no superelastic like reversion upon unloading in the curves of those alloys. In the curves of Ti5Mo-4 and 5Sn alloys, the superelastic like reversion was clearly recognized upon unloading. This indicates the instability of stress induced martensite in both the alloys resulting from the lowering of transformation temperatures by $\mathrm{Sn}$ addition. Therefore, it is suggested that the $R_{\mathrm{sb}}$ relates to the potentiality of superelasticity. In fact, we have succeeded to obtain the nearly perfect superelasitic recovery of $3.5 \%$ tensile strain in the aged Ti-5Mo-5Sn alloy at room temperature, which will be reported soon.

Figures 3(a) and (b) show XRD profiles of Ti-5Mo-Ag and Ti-5Mo-Sn alloys, respectively. $\beta$ phase and small amount of $\alpha^{\prime \prime}$ martensitic phase are identified in Ti-5Mo binary alloy. The amount of $\alpha^{\prime \prime}$ martensite phase increases in Ti-5Mo-1 Ag and then decreases in Ti-5Mo-3Ag and Ti-5Mo-5Ag. This result might be explained as follows. It is expected that both $\beta$ and martensitic transformation temperatures are reduced by addition of $\mathrm{Ag}$, because $\mathrm{Ag}$ is $\beta$ stabilizing element as mentioned above. Contrary to the expectation, the experimental results in Fig. 3(a) show opposite tendency. In such case, the mutual effect of athermal $\omega$ phase formation and solid solution strengthening of $\beta$ phase by $\mathrm{Ag}$ should be considered. The athermal $\omega$ phase usually suppresses the martensitic transformation. The amount of athermal $\omega$ phase is decreased by the addition of $\mathrm{Ag}$ as a result of stabilization of $\beta$ phase. Consequently, $M_{\mathrm{s}}$ temperature is increased. Solid solution strengthening of $\beta$ phase by $\mathrm{Ag}$ prevents the lattice distortion of martensitic transformation. From these consideration, the increase of $\alpha^{\prime \prime}$ martensitic phase in Ti-5Mo-1Ag is attributable to the decrease of the amount of athermal $\omega$ phase and the decrease of that in $\mathrm{Ti}-5 \mathrm{Mo}-3$ and $5 \mathrm{Ag}$ is attributable to the prevention of lattice distortion due to the solid solution strengthening of Ag. In the case of Ti-Mo-Sn alloys, $\beta$ and martensitic transformation temperatures should be increased by addition of $\mathrm{Sn}$, because $\mathrm{Sn}$ is $\alpha$ stabilizing element as mentioned above. However, only $\beta$ phase is identified in Ti-5Mo-1 to 5Sn and no $\alpha^{\prime \prime}$ martensitic peak is detected as shown in Fig. 3(b). This result might be explained by mutual balance between the decrease of athermal $\omega$ phase formation and sold solution strengthening by $\mathrm{Sn}$ as well as $\mathrm{Al}$ in Ti-Mo- $\mathrm{Al}$ alloys. ${ }^{7} \mathrm{Al}$ is also typical $\alpha$ stabilizing element. In Ti-Mo-Al alloys, the $M_{\mathrm{s}}$ temperature increases with decreasing the athermal $\omega$ phase formation in low Al alloys and decreases with increasing the solid solution strengthening of $\mathrm{Al}$ in high $\mathrm{Al}$ alloys. Therefore, the solid solution hardening is considered to be more dominant than the decrease of athermal $\omega$ phase formation in the present Ti5Mo-Sn alloys. This is consistent with the recent result of Ti$\mathrm{Nb}-\mathrm{Sn}$ alloys. It has been reported that $1 \%$ increase in $\mathrm{Sn}$ content lowers martensitic transformation temperatures by about $150 \mathrm{~K}$ in $\beta$-type Ti-Nb-Sn alloy. ${ }^{4}$ 

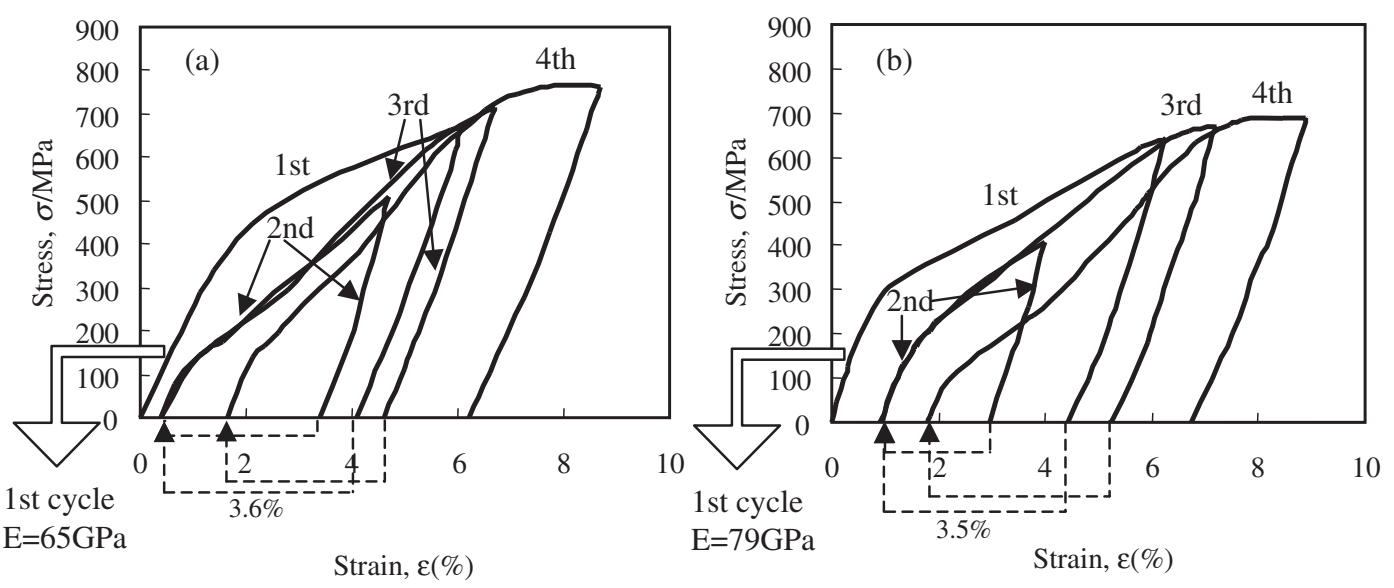

Fig. 4 Stress-strain curves obtained from cyclic tensile test at room temperature. Broken lines indicate shape recovery with heating. (a) Ti-5Mo-4Ag and (b) Ti-5Mo-3Sn alloys.
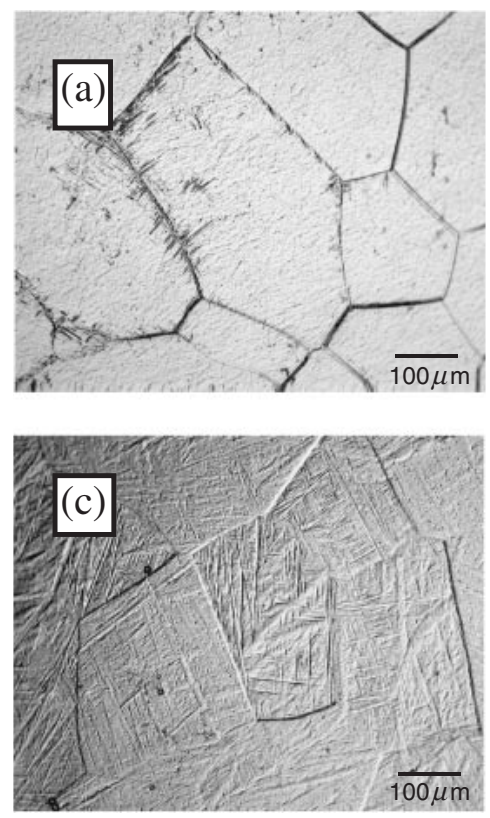

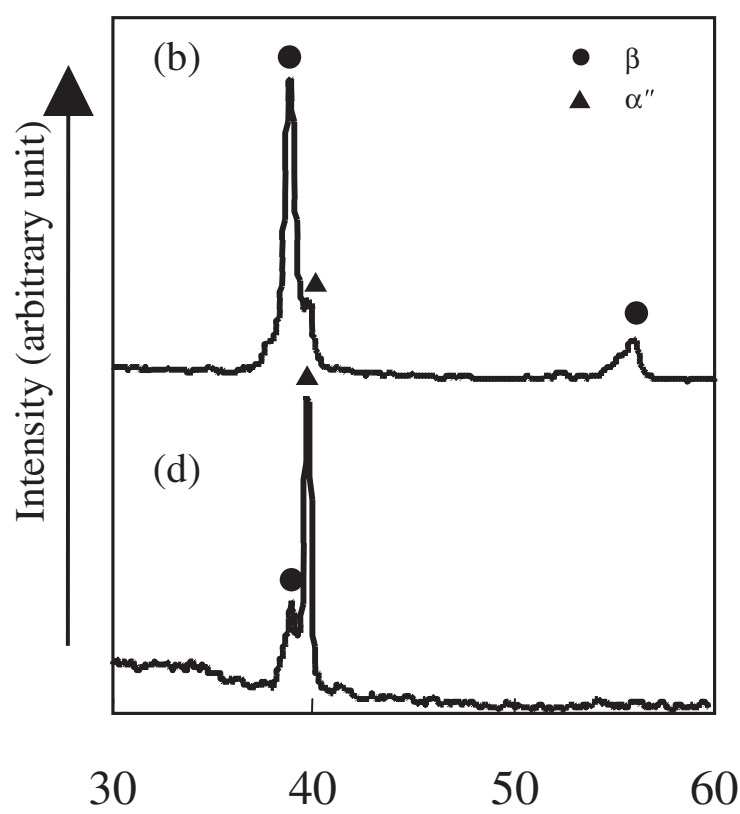

Diffraction angle, $2 \theta$

Fig. 5 Optical micrographs and XRD profiles in Ti-5Mo-4Ag alloy, (a) and (b) before, and (c) and (d) after tensile deformation with 4\% strain.

Figures 4(a) and (b) show stress-strain curves measured through cyclic tensile deformation of Ti-5Mo-4Ag and Ti$5 \mathrm{Mo}-3 \mathrm{Sn}$ alloys, respectively. Broken lines with arrows indicate the shape recovery with heating. The maximum shape recovery strain in the Ti-5Mo-4Ag alloy is $3.6 \%$ after the first cycle and that in the Ti-5Mo-3Sn alloy is $3.5 \%$ after the first cycle. Young's modulus of Ti-5Mo-4Ag is determined to be $65 \mathrm{GPa}$ by the strain gage method. That of Ti$5 \mathrm{Mo}-3 \mathrm{Sn}$ is $79 \mathrm{GPa}$. These young's modulus values are lower than that of $\mathrm{Ti}$ which is $106 \mathrm{GPa}^{8}{ }^{8}$ It is suitable for the biomedical material. The yield stress abruptly decreases at the second cycle in both the alloys. In the Ti-5Mo-4Ag alloy the yield stress at the fourth cycle slightly increases. On the other hand, in the Ti-5Mo-3Sn alloy the yield stress at the fourth cycle further decreases. The same phenomena have been reported in Ti-Mo-Al and Ti-Mo-Ga alloys. ${ }^{2,3)}$ It might be due to mutual effect of slip deformation in $\beta$ phase, stress induced martensitic transformation and reorientation of martensite variants with loading, since the stress required for reorientation of martensite variants is usually lower than that for the rest in shape memory alloys. The actual mechanism is now under study and it will be reported in due course. However, the yielding at the second and third cycles is due to stress induced martensitic transformation and/or reorientation of martensite variants because of the complete shape recovery at the second cycle in the both alloys. It is estimated that the reliable shape recovery strain in both the alloys is about $3 \%$. Therefore, it can be concluded that Ti-Mo-Ag and Ti-Mo-Sn alloy systems are promising shape memory materials. Further research is required to 
improve the shape memory properties through microstructure control with various heat treatments in order to replace TiNi alloys in the biomedical field.

Microstructure and XRD profile changes in Ti-5Mo-4Ag alloy before and after tensile deformation are shown in Fig. 5. There are $\beta$ phase and a small amount of $\alpha^{\prime \prime}$ martensitic phase before the deformation as shown in Figs. 5(a) and (b). After the bending deformation with $4 \%$ strain banded surface relief of $\alpha^{\prime \prime}$ martensitic phase appears as shown in Fig. 5(c). This is supported by XRD profiles in Fig. 5(d) in which the intensity of $\beta$ phase decreases and that of $\alpha^{\prime \prime}$ phase increases remarkably. Similar results were obtained in Ti-5Mo-3Sn alloy. It is clear that the stress induced $\beta$ to $\alpha^{\prime \prime}$ martensitic transformation associates with the shape memory effect in both the alloys.

\section{Conclusions}

Ti-Mo-Ag and Ti-Mo-Sn alloys for biomedical applications are developed and their shape memory properties are investigated. The results are summarized as follows.

(1) The shape memory effect is obtained in most of the prepared alloys and $\mathrm{Ti}-5 \mathrm{Mo}-2$ to $5 \mathrm{Ag}$ and $\mathrm{Ti}-5 \mathrm{Mo}-1$ to 3Sn alloys exhibit the best performance.

(2) A maximum shape recovery strain in the Ti-5Mo- $4 \mathrm{Ag}$ alloy is $3.6 \%$ after the first cycle and that in the Ti-5Mo$3 \mathrm{Sn}$ alloy is $3.5 \%$ after the first cycle. It is estimated that the reliable shape recovery strain in both the alloys is about $3 \%$.
(3) Microstructure and XRD profile changes before and after tensile test revealed that the stress induced $\beta$ to $\alpha^{\prime \prime}$ martensitic transformation played an important role of the shape memory effect in both the alloys.

\section{REFERENCES}

1) SA Shabalovskaya: Inter. Mater. Rev. 46 (2001) 233-250.

2) H. Hosoda, N. Hosoda and S. Miyazaki: Trans. MRSJ 26 (2001) 243246.

3) H. Hosoda, Y. Ohmatsu and S. Miyazaki: Trans. MRSJ 26 (2001) 235237.

4) E. Takahashi, T. Sakurai, S. Watanabe, N. Masahashi and S. Hanada: Mater. Trans. 43 (2002) 2978-2983.

5) D. Kuroda, M. Niinomi, M. Morinaga, Y. Kato and T. Yashiro: Mater. Sci. Eng. A243 (1998) 244-249.

6) Materials Properties Handbook: Titanium alloys, ed. by ASM International (1994) 7-9.

7) M. Ikeda, S. Komatsu and T. Sugimoto: JJILM 50 (2000) 435-440.

8) Metal Data Book, ed. by Jpn. Inst. Mat., (Maruzen. Co., Ltd., 1993) 31

9) H. Ohyama and T. Nishimura: Proc. 8th world Conf. on Titanium (1995) pp. 2273-2280.

10) T. Furuhara, T. Makino, Y. Idei, H. Ishigaki, A. Takada and T. Maki: Mater. Trans., JIM 39 (1998) 31-39.

11) C. Y. Lei, J. S. Lee Pak, H. R. P. Inoue and C. Marvin Wayman: Proc. Int. Conf. on Martensitic Transformation (1992) pp. 539-544.

12) H. Sasano and T. Suzuki: Proc. 5th Int. conf. of Titanium (1984) pp. 1667-1674.

13) T. Hamada, T. Sodeoka and M. Miyagi: Proc. 6th world Conf. of Titanium (1988) pp. 877-889.

14) T. Sugimoto, M. Ikeda, S. Komatsu, K. Sugimoto and K. Kamei: Proc. 6th world Conf. of Titanium (1988) pp. 1069-1074. 